\title{
Spatial and Electronic Manipulation of Silicon Nanocrystals by Atomic Force Microscopy*
}

\author{
L. Douglas Bell \\ Jet Propulsion Laboratory, Caltech, Pasadena, CA \\ Phone: 818/354-4761 Email: dbell@vaxeb.jpl.nasa.gov \\ Deborah H. Santamore, Elizabeth A. Boer, \\ Harry A. Atwater, Kerry J. Vahala and Richard C. Flagan \\ Thomas J. Watson Lab. Of Applied Physics, California Institute of Technology, \\ Pasadena, CA
}

\begin{abstract}
As silicon-based devices shrink, interest is increasing in fast, low-power devices sensitive to small numbers of electrons. Recent work suggests that MOS structures with large arrays of Si nanocrystals comprising a floating gate can be extremely fast, reliable and nonvolatile relative to conventional floating gate memories. In these structures approximately one electron is stored per nanocrystal. Despite promising initial results, current devices have a distribution of charge transit times during writing of nanocrystal ensembles, which limits speed. This behavior is not completely understood, but could be related to a dispersion in oxide thicknesses, nanocrystals interface states, or shifts in the electronic bound states due to size variations. To address these limitations, we have developed an aerosol vapor synthesis/ deposition technique for silicon nanocrystals with active size classification, enabling narrow distributions of nanocrystal size ( $\sim 10-15 \%$ of particle in the $2-10 \mathrm{~nm}$ size range).
\end{abstract}

The first goal of these experiments has been to use scanning probe techniques to perform particle manipulation and to characterize particle electronic properties and charging on a singleparticle basis. Si nanocrystal structures (lines, arrows and other objects) have been formed by contact-mode operation and subsequently imaged in noncontact mode without additional particle motion. Further, single nanocrystal charging by a conducting AFM tip has been observed, detected as an apparent height change due to electrostatic force, followed by a slow relaxation as the stored charge dissipates. Ongoing and future efforts will also be briefly discussed, including narrowing of nanocrystal size distributions, control of oxide thickness on the nanocrystals, and measurements of electron transport through individual particles and ensembles.

* Rescarch supported by JPL DRDF and NASA 